\title{
Novel bilateral bifurcation of the coronary vasculature
}

\author{
Adam Michael Taylor ${ }^{1}$, Joe McAleer ${ }^{2}$, Quenton Wessels ${ }^{3}$ \\ ${ }^{1}$ Clinical Anatomy Learning Centre, Lancaster Medical School, Faculty of Health and Medicine, Lancaster University, Lancaster, ${ }^{2}$ Royal Preston \\ Hospital, Fulwood, United Kingdom, ${ }^{3}$ Department of Anatomy, School of Medicine, University of Namibia, Windhoek, Namibia
}

\begin{abstract}
Multiple variants and anomalies in the coronary vasculature have been reported. Some variants, particularly those with duplication, can be advantageous, many are insignificant and some are ultimately lethal. Many of these variants and anomalies are not identified until imaged or post-mortem. A novel bilateral bifid variation of the coronary arteries was observed in 49-year-old male cadaver. The respective origins were associated with the left and right aortic cusps from single ostia. Immediate bifurcation followed on either side. A right sided accessory branch supplied the pulmonary trunk and right ventricle. A more standard branch continued to form the posterior interventricular artery. The left sided variation demonstrated a solitary circumflex artery (towards the posterior interventricular septum) and, left marginal and anterior interventricular branches shortly after bifurcation. This case may be beneficial in surviving adverse cardiac events, particularly those associated with lifestyle. They also present a 'double' challenge for clinicians.
\end{abstract}

Key words: Anatomy; Coronary vessels; Variation; Embryology

Received September 12, 2020; Revised October 28, 2020; Accepted December 14, 2020

\section{Introduction}

The typical two coronary artery system supplying the heart was first described by Riva di Trento in 1559. The significance of the vessels and associated pathological conditions would become clear in the subsequent centuries. Notably, in 1768 British Physician William Heberden first described Angina Pectoris. This description would be used by Edward Jenner to make the first documented description between the symptoms of Angina Pectoris and its causecoronary artery disease [1].

Variations in the coronary vasculature can either be harmless or pose a serious problem when considering di-

\section{Corresponding author:}

Ouenton Wessels (iD

Department of Anatomy, School of Medicine, University of Namibia, Private Bag 13301, Windhoek, Namibia

E-mail:qwessels@unam.na agnostic and therapeutic procedures [2, 3]. Furthermore, anomalies in the coronary vasculature are linked with sudden death syndromes in the young, and defining these better may prove helpful in screening programmes [4].

In this case report, we describe an extremely rare and novel coronary variant. Understanding variations and their prevalence may lead to better clinical outcomes, reduced surgical time and increased options where bypass graft surgery is needed.

\section{Case Report}

Anatomical variations of the coronary arteries were observed during routine dissection of a 49-year-old male formalin embalmed cadaver from the Western Cape, South Africa. The cadaver was kindly donated to the Department of Anatomy, University of Namibia. The cause of death was documented as natural causes and no gross pathologies, such atherosclerotic plaques or coronary heart disease, were found. Both the left and right coronary arteries (LCA and 
RCA) arose superior to the left and right aortic cusps respectively (Fig. 1A, B, 1 and 2). The luminal diameter of the LCA at its origin was $3.47 \mathrm{~mm}$ and that of the RCA was $3.35 \mathrm{~mm}$ (Fig. 1B, asterisks). Both arteries bifurcated shortly thereafter (Fig. 1A, B, 1 and 2) forming distinctive 'shotgun' orifices. At the LCA one was positioned anteriorly and the other posteriorly with luminal diameters of $3.33 \mathrm{~mm}$ and $3.21 \mathrm{~mm}$ respectively (Fig. 1B). The two ostia of the RCA were positioned medial and lateral with luminal diameters of $3.05 \mathrm{~mm}$ and $2.47 \mathrm{~mm}$ respectively (Fig. 1B). An accessory right coronary artery (Fig. 1A-C, 5) was formed - anterior and parallel to its traditional counterpart (Fig. 1A-C, 4). The anterior accessory branch was found to supply the pulmonary trunk (Fig. $1 \mathrm{~A}, \mathrm{C}, 7)$ and the right ventricle (right ventricular branch). It also continued to form the right marginal artery - extending towards the apex of the heart. The more conventional right coronary artery continued to descend and form the posterior interventricular branch after coursing within the coronary sulcus (Fig. 1A, C, 4). On the left, a solitary circumflex artery (luminal diameter $=2.89 \mathrm{~mm}$ ) continued towards the poster interventricular septum shortly after the bifurcation of the left coronary artery (Fig. 1A, D, 3). The circumflex branch also supplied the left atrium. A more traditional pattern followed after bifurcation with the establishment of the anterior interventricular artery, hereafter referred as LAD (left anterior descending artery) (Fig. 1A, D, 9) and a left marginal artery (Fig. 1A, D, 10) with luminal diameters of $3.64 \mathrm{~mm}$ and $2.66 \mathrm{~mm}$ respectively.

\section{Discussion}

The coronary vessels are typically seen as a pair of structures that arise from the ascending aorta. The origin of the left and right coronary arteries is usually from the left posterior aortic sinus (LPAS) and the anterior aortic sinus (AAS), respectively. The lumina of the ostia are typically $3.6 \mathrm{~mm}$
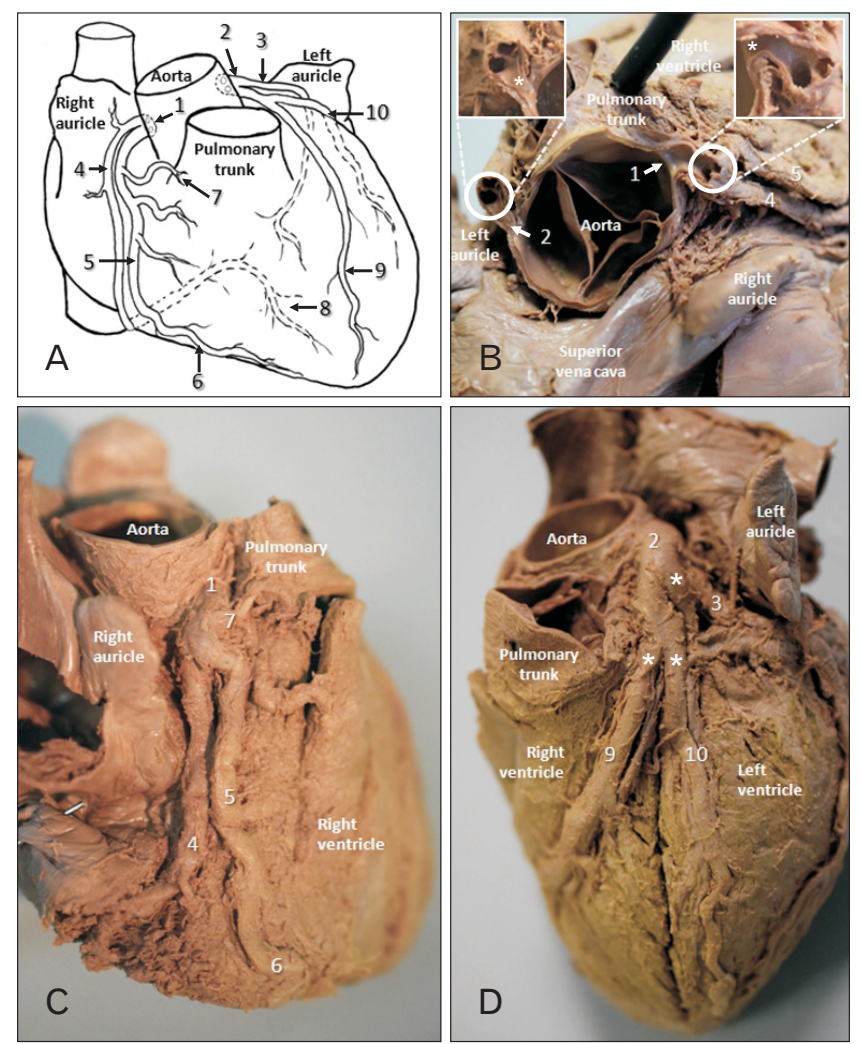

Fig. 1. The distribution of the coronary arteries. (A) Anterior view schematic. (B) Superior resected view with expanded captions of 'shotgun' orifices after bifurcation (asterisks indicating the origins of the coronary arteries). (C) Right anterior oblique view. (D) Left anterior oblique view (asterisks indicating the measurement points of the luminal diameters). 1, right coronary artery; 2, left coronary artery; 3, circumflex artery (leading towards the posterior interventricular septum); 4, right coronary artery (also supplying the SA node); 5 accessory right coronary (supplying the pulmonary trunk and providing a right ventricular branch); 6, right marginal artery; 7, branch to the pulmonary trunk; 8, PDA; 9, LAD; 10, left marginal artery; LAD, anterior interventricular artery; PDA, posterior interventricular artery; SA, sinoatrial. 
(R) and $4.1 \mathrm{~mm}(\mathrm{~L})$ in diameter at their origins, ours were smaller than the average reported [4].

Duplicate ostia usually indicate conus artery presence, which has earnt the conus artery the title 'the third coronary artery' in some texts. There are reports of up to five ostia being present across the AAS and LPAS combined [5]. The typical presentation of multiple ostia in the AAS sees the RCA and conus artery arising separately, whereas in the LPAS multiple ostia are usually the LAD and left circumflex arteries arising separately, with no left coronary artery as a standalone vessel.

Documented variations of the coronary vessels, such as coronary dominance and high take-off of the arteries from the aorta; are all considered within a range of normal variation. Coronary artery anomalies in adults are usually incidental observations in imaging studies or cadavers, deemed as such if it is seen in less than $1 \%$ of the population [6]. Coronary artery anomalies can be classified clinically into benign and malignant types in terms of their potential for sudden death. There are cases in the literature that describe a number of coronary artery anomalies, such as: anomalous course, origin, or both [7]. Regarding anomalies in the origin of the vessels, there are cases showing duplicate vessels from a single aortic sinus [8]. There are four common anomalous variants of the origins of coronary vessels $[6,8]$;

1) the RCA arising from the same sinus as the LCA, the LPAS,

2) the LCA arising from the same sinus as the RCA, the AAS,

3) the left circumflex, or LAD arising from the AAS,

4) either coronary artery arising from the non-coronary sinus.

Where these variants arise, they have common courses that supply the relevant areas of myocardium; 1) between the aorta and pulmonary artery, 2) retro-aortic, 3) in front of the pulmonary artery, 4) beneath the pulmonary artery.

The relationship of the anomalous artery to the outflow tracts from the heart is clinically significant as those with a course between the aorta and the pulmonary artery are at an increased risk of death. Studies show cardiac structural abnormalities were documented in over $50 \%$ of 124 nontraumatic cases of sudden death, of those 64 , over $60 \%$ had coronary artery abnormalities [9]. These sudden deaths occur with the onset of, or shortly after, vigorous exercise and in individuals who are younger than 35 yrs of age. The most common coronary anomaly seen was the presentation of a coronary artery from the opposite sinus. Where the LCA is seen arising from the anterior (right) sinus resulting in it coursing between the pulmonary artery and the aorta, potentially increasing pressure on the LCA between the two major vessels during exercise. A combination of both, increased diastolic expansion of the great vessels and an increasing cardiac output leads the aberrant vessels to be unable to match the myocardial oxygen demand of the heart [10, 11]. This leads to myocardial ischaemia, which may present as a sudden death either due to an acute ischaemic event or the summation of multiple episodes.

In rare instances $(0.0024 \%-0.044 \%$ of the population), both coronary vessels can arise from a single ostium in a single sinus [8].

These anomalies tend to be asymptomatic, but nonetheless the eventual threat to life does render them malignant anomalies. Some variants in origin are overtly symptomatic in infancy, such as the Bland-White-Garland syndrome, also known as anomalous origin of the left coronary artery from the pulmonary artery where the LCA originates from the pulmonary trunk. This is apparent in $0.25 \%-0.5 \%$ of congenital cardiac abnormalities with a mortality in infants of $90 \%$, due to coronary steal syndrome [11]. There are rarer still reports of a similar anomaly of the RCA [12].

There is not always a clear distinction between variance and anomalies in regards to coronary artery anatomy. There is a degree of variance in LAD myocardial tunnelling or myocardial bridging. This is where the course of the artery burrows down into the myocardium for a part of its length. It's been reported that in up to $70 \%$ of autopsies presented myocardial strands that surround CAs, regardless of the cause of death, with true LAD myocardial bridging being prevalent in approximately $26 \%$ of cases [13]. In such regard, myocardial bridging should be seen as a variant. However, there is evidence of some pathological component to this as a tunnelled LAD has been seen in $5 \%$ of sudden deaths in young athletes, and has symptomatic associations with angina pectoralis and myocardial infarction in the population at large. Indeed, stenting or coronary artery bypass grafting of the tunnelled portion of the LAD for severe disease, has had excellent symptomatic control [14].

During embryological development a capillary plexus, formed by angioblasts, exists over the early heart. At the most proximal ends, the ostia form imminently after truncal separation. Distally the vessels remain part of superficial plexus awaiting growth of the cardiac muscle. As develop- 
ment proceeds, the plexus remodels forming the vessels, this remodelling may result in duplicate, or anomalous vessels, with hypoxia driving the process.

Duplicate or parallel coronary vessels are incredibly rare, with only a small number of cases of double right coronary arteries [15] and absence of double left coronary arteries from the literature. It is entirely possible that having a double supply to an area of myocardium maybe beneficial, providing an alternative for when one may become occluded. When sudden death in younger individuals is observed, coronary vasculature may be a potentially contributing factor. To the authors knowledge ours is the first documented case of bilateral bifid/duplicate coronary arteries.

\section{ORCID}

Adam Michael Taylor:

https://orcid.org/0000-0002-4084-0456

Joe McAleer: https://orcid.org/0000-0002-2377-0978

Quenton Wessels: https://orcid.org/0000-0001-8539-5459

\section{Author Contributions}

Conceptualization: AMT, JM, QW. Data acquisition: JM. Data analysis or interpretation: AMT. Drafting of the manuscript: AMT. Critical revision of the manuscript: QW. Approval of the final version of the manuscript: all authors.

\section{Conflicts of Interest}

No potential conflict of interest relevant to this article was reported.

\section{References}

1. Quinn T. The history of cardiac care. In: Barrett D, Gretton M, Quinn T, editors. Cardiac Care: An Introduction for Healthcare Professionals. Chichester: John Wiley \& Sons; 2006. p.10-8.

2. Nayak SB. Trifurcation of right coronary artery and its huge right ventricular branch: can it be hazardous? Anat Cell Biol
2018;51:139-41.

3. Prakken NH, Cramer MJ, Olimulder MA, Agostoni P, Mali WP, Velthuis BK. Screening for proximal coronary artery anomalies with 3-dimensional MR coronary angiography. Int J Cardiovasc Imaging 2010;26:701-10.

4. Pejković B, Krajnc I, Anderhuber F. Anatomical variations of coronary ostia, aortocoronary angles and angles of division of the left coronary artery of the human heart. J Int Med Res 2008;36:914-22.

5. Waller BF. Five coronary ostia: duplicate left anterior descending and right conus coronary arteries. Am J Cardiol 1983;51:1562.

6. Villa AD, Sammut E, Nair A, Rajani R, Bonamini R, Chiribiri A. Coronary artery anomalies overview: the normal and the abnormal. World J Radiol 2016;8:537-55.

7. Basso C, Thiene G. Congenital coronary artery anomalies at risk of myocardial ischaemia and sudden death. Eur Cardiol 2005;1:1-5.

8. Gräni C, Kaufmann PA, Windecker S, Buechel RR. Diagnosis and management of anomalous coronary arteries with a malignant course. Interv Cardiol 2019;14:83-8.

9. Illes J, Moser MA, McCormick JB, Racine E, Blakeslee S, Caplan A, Hayden EC, Ingram J, Lohwater T, McKnight P, Nicholson C, Phillips A, Sauvé KD, Snell E, Weiss S. Neurotalk: improving the communication of neuroscience research. Nat Rev Neurosci 2010;11:61-9.

10. Frescura C, Basso C, Thiene G, Corrado D, Pennelli T, Angelini A, Daliento L. Anomalous origin of coronary arteries and risk of sudden death: a study based on an autopsy population of congenital heart disease. Hum Pathol 1998;29:689-95.

11. Rahalkar AM, Rahalkar MD. Pictorial essay: coronary artery variants and anomalies. Indian J Radiol Imaging 2009;19:4953.

12. Rama Krishnan RS, Marwah VV, Gupta T, Kalyanpur A. Images: malignant right coronary artery- 64-slice CTA. Indian J Radiol Imaging 2008;18:126-7.

13. Risse M, Weiler G. [Coronary muscle bridge and its relations to local coronary sclerosis, regional myocardial ischemia and coronary spasm. A morphometric study]. Z Kardiol 1985;74:700-5. German.

14. Lee MS, Chen CH. Myocardial bridging: an up-to-date review. J Invasive Cardiol 2015;27:521-8.

15. Sari I, Kizilkan N, Sucu M, Davutoglu V, Ozer O, Soydinc S, Aksoy M. Double right coronary artery: report of two cases and review of the literature. Int J Cardiol 2008;130:e74-7. 